\title{
APPLICATION AND DEVELOPMENT OF LED DISPLAY IN SPORTS FIELD
}

\author{
Jing LIU \\ Beihang University, Department of Physical Education, Beijing, 100191, China; \\ E-mail: irxcva78961171@126.com
}

\begin{abstract}
LED screens have become a must-have for modern large-scale sports venues and are indispensable equipment for major sports events. Therefore, the application and development of LED display in the field of sports are studied. The LED dot matrix is selected as the display system of the automatic track circle system of the sports track competition. By analyzing the display principle of the display screen pair code and Chinese characters applied in the sports field, an algorithm is proposed to develop the application of the display screen in the sports field for the sports field. The application display provides a reference for character and Chinese character display. The debugging result is consistent with the expected goal, and the number of displays and the number of additions and subtractions can be realized by keyboard operation, and the remaining number of laps of the athlete is correctly displayed.

Keywords: LED display, sports field, development research
\end{abstract}

\section{INTRODUCTION}

After entering the $21^{\text {st }}$ century, as one of the main information distribution media, the LED display industry and technology have made some progress [1]. Its use in the sports industry is to serve sports competitions. Therefore, in product research and development, it should first meet the requirements of sports competitions, and can play the role of timing scoring in sports competitions, and timely broadcast the contestants' competition results and related materials, which is the main role that LED display screens can enter the sports industry at the beginning. In addition, it has a strong rendering effect in the sports game highlight playback, real-time broadcast, background image, etc., and can create a very lively atmosphere [2]. The LED display panel is used as a display method for controlling semiconductor light emitting diodes to display a series of information such as text, graphics, images, animations, quotes, videos, etc. [3]. Compared with other terminal displays, it has the advantages of high brightness, long life cycle, large visual range, easy interface, and support software [4]. It has gradually become one of the must-have facilities for contemporary large stadiums, and the use of more LED screens in important sports is an indispensable device. The display system of the stadium should be able to display the event information clearly and correctly, and use multimedia technology to show the situation of the competition and render the atmosphere of the scene. Therefore, the paper has a very important practical value for the use and development of LED display in the sports industry.

\section{STATE OF THE ART}

Western exploration of LEDs is early, generally analyzing the materials, colours, and brightness of LEDs. China's more well-known enterprises include Shenzhen Ai Biesen and Huizhou Desai. Compared with Western countries, domestic research is biased. Late, it is basically the exploration of LEDs by the Chinese Academy of Sciences and some schools. However, the countries that widely use 
LED displays are basically in Asia, such as China, South Korea, Japan, etc., and Europe and the United States [5]. Along with the continuous advancement of LED technology, a large number of stadiums have replaced LEDs and CRT displays with LED displays, and the displayed information has been converted from past figures into text, images, and videos [6]. The LED display of the stadium serves as the main means of game information display and live broadcast of the game. It is connected with the game's timing and scoring system, plays the player's game results and related materials, publishes sports information, and displays text animation and video images, which is one of the most important information dissemination vehicles on the scene and is the "soul" device in many facilities of the stadium [7]. In the $43^{\text {rd }}$ World Table Tennis Championships held in Tianjin, China in 1995, a huge LED screen of more than 1,000 square meters is used. At the opening ceremony of the Beijing Olympic Games in 2008, the dream world created by LED and projector has already amazed the audience. At present, the high-standard stadium LED display has gradually become one of the necessary facilities, and the demand for LED screens in the stadium has been increasing. At this stage, the design technology, control technology, manufacturing process and usage level of domestic LED display screens are close to international standards, and the production cost is significantly less than that of international products [8].

\section{METHODOLOGY}

\subsection{Gray-Scale Modulation Model Algorithm Based on LED Display in Sports Field}

Generally speaking, indoor gymnasiums must have one or two LED displays installed, which can be hung on the wall. The display should be serviced by sports competitions, which can be seen by most people. It is assumed that the venues are large in scale and there are more seats in the audience. Then, it is possible to install a number of combined display screens in the central part of the venue. For example, the Wukesong Basketball Hall in Beijing uses a multi-piece combination display system. The whole system consists of a "funnel screen", a ring screen and a timing score control system. Among them, the display screen is installed in the central part of the venue weighs 22 tons. Because it
Table 1. Determination of the Size of the Frame of the Stadium LED Display

\begin{tabular}{|c|c|}
\hline $\begin{array}{l}\text { Stadium audience } \\
\text { capacity }\end{array}$ & LED display frame size \\
\hline 1,0000 seats or less & $\begin{array}{l}\text { 3000mm(height) } \times 10000 \mathrm{~mm} \\
\text { (length) }\end{array}$ \\
\hline 2000 3000 seats & $\begin{array}{c}5000 \mathrm{~mm} \text { (height) } \times 15000 \mathrm{~mm} \\
\text { (length) }\end{array}$ \\
\hline $3000 \sim 4000$ seats & $\begin{array}{c}\text { 7000(height) } \times 20000 \mathrm{~mm} \\
\text { (length) }\end{array}$ \\
\hline $\begin{array}{l}\text { More than } 8,000 \\
\text { seats }\end{array}$ & $\begin{array}{l}\text { 8000(height) } \times 25000 \mathrm{~mm} \\
\text { (length) }\end{array}$ \\
\hline
\end{tabular}

looks like a funnel, it is called a "funnel screen." It is divided into five layers, with a net height of 9.042 meters and a maximum diameter of 11 meters. Generally speaking, only one LED display is installed in the outdoor venue. It is more reasonable to set it above the southern stands, facing the north, so as to prevent direct sunlight from affecting the effect. The display of the outdoor venue has high requirements for brightness. In the direct sunlight, the audience needs to see the content above. Similarly, it should have strong wind, defence, and anti-corrosion and lightning protection capabilities. The interior has good ventilation and heat dissipation. The size should be determined according to the size of the site, the space and the audience capacity. In addition, the venue is brighter and the viewing range is larger. Therefore, the specifications and brightness of the LED screen should be clearly defined according to the actual situation, which refers to Table 1 for specific settings.

The number of pixels of the LED display, $N_{L E F}$, can be calculated from the width and height of the display for:

$$
N_{L E F}=N_{w} \times N_{h}
$$

Among them, $N_{w}$ represents the width of the display screen in the model, and $N_{h}$ represents the height of the display screen in the model. From the perspective of the driver chip, the number of pixels of the LED display is $N_{L E F}$. It can also be expressed as:

$$
N_{L E F}=N_{i c} \times N_{c h}
$$


Table 2. Proportion of Brightness Loss Within of the Reference Time

\begin{tabular}{|c|c|c|c|c|c|}
\hline $\begin{array}{c}\Delta N_{\text {latch }} \\
N_{\text {latch }}\end{array}$ & 1 & 2 & 3 & 4 & 5 \\
\hline 32 & 3.03 & 5.88 & 8.57 & 11.11 & 13.51 \\
\hline 64 & 1.54 & 3.03 & 4.48 & 5.88 & 5.88 \\
\hline 96 & 1.03 & 2.04 & 3.03 & 4.00 & 7.25 \\
\hline 128 & 0.78 & 1.54 & 2.29 & 3.03 & 4.95 \\
\hline 192 & 0.52 & 1.03 & 1.54 & 2.04 & 3.76 \\
\hline 256 & 0.39 & 0.77 & 1.16 & 1.54 & 1.91 \\
\hline
\end{tabular}

Wherein, $N_{i c}$ represents the number of driving chips used for one channel of data, and $N_{c h}$ represents the number of channels included in each driving chip. If the latch length is defined as $N_{\text {Iatch }}$, obviously:

$$
N_{\text {Iatch }}=N_{i c} \times N_{c h} \text {. }
$$

For a data link with a length of $N_{\text {Iatch }}$, the time required for the migration is $\tau_{\text {Iatch }}$ :

$$
\tau_{\text {Iatch }}=\frac{1}{f_{\text {clk }}} \times N_{\text {Iatch }} \text {. }
$$

In actual engineering use, there will be some idle clocks in the gap between each two shift operations. The shift gap is used for timing adjustment, which is recorded as $\Delta N_{\text {Iatch }}$, so the time required to transfer data once is $\tau_{0}$, which is expressed as:

$$
\tau_{0}=\frac{1}{f_{c l k}}\left(N_{\text {Iatch }}+\Delta N_{\text {Iatch }}\right) .
$$

Here $\tau_{0}$ is defined as the reference time of the scan, also known as a time slice, which is an important parameter based on the time slice gray modulation. It can be seen that in the case where the scan clock is constant, the reference time increases as the length of the shift increases. If the reference time is kept constant, the scan clock needs to increase as the length of the shift increases. The latch signal is a very narrow level signal, and the number of scan clocks is recorded as $N_{L E}$, which is a part of the middle of $\Delta N_{\text {Iatch }}$. During the benchmark time, the bandwidth utilization and bandwidth loss rates are:

$$
\left\{\begin{array}{l}
\eta_{B L}=\frac{N_{\text {latch }}}{N_{\text {latch }}+\Delta N_{\text {latch }}} \times 100 \% \\
\eta_{\Delta B L}=\frac{N_{\text {latch }}}{N_{\text {latch }}+\Delta N_{\text {latch }}} \times 100 \%
\end{array} .\right.
$$

Table 2 shows the ratio of luminance loss in the reference time. The first behaviour is the shifting gap. The range is from 1 to 5 . The first column is the shifting length. The range is from 32 to 256 . The other parts are the luminance loss during the reference time. It can be seen that when the transfer length is constant, the brightness loss in the reference time increases with the increase of the transfer gap; when the transfer gap is constant, the brightness loss decreases as the transfer length increases. In practical engineering applications, the transfer gap is generally set to be adjustable, in order to obtain better bandwidth utilization, if there is no special explanation about the calculation of the transfer gap mentioned later, take $N_{\text {Iatch }}=3$, at this time, if the transfer length is 256, the bandwidth loss rate is $1.16 \%$ in the reference time. If the gray level of the display screen is $n$ bit, the single primary colour image data is represented as $\mathrm{D}[(\mathrm{n}-1): 0]$, and each bit of data is represented as $\mathrm{D}[\mathrm{n}-1], \mathrm{D}[\mathrm{n}-2], \ldots, \mathrm{D}[1]$, $\mathrm{D}[0]$. During the reference time, the relationship between the stored serial data sequence $\mathrm{D}[\mathrm{x}]$ and the data bits can be expressed as:

$$
D[x]=\bigcup_{i=0}^{N_{L E D}-1} D_{i}[x],(0 \leq x<n) .
$$

From equation 7 , when $\mathrm{x}=0, D[0]=\left\{D_{0}[0]\right.$, $\left.D_{1}[0], \ldots, D_{N_{L E D-1}}[0]\right\}$, the serial data that is sequentially transferred in a time slice is indicated. 


\subsection{Time Matching Algorithm}

The core of the LED display is the scan clock. The scan clock is like the human blood, driving all the display logic. This section expresses the relationship between the gray parameters based on the scan clock and gray level. In order to realize the gray level modulation of the LED display screen and obtain the preferred scanning clock, according to the gray level modulation model, the gray level modulation process is divided into basic gray level modulation and extended gray level modulation, and an optimization is proposed by analyzing the relationship between the parameters. The selection method of scan clock and the concept of scan clock redundancy are summarized on the basis of this. Finally, the gray level model is verified and implemented by the hardware platform. According to the grayscale modulation model, the grayscale modulation process is divided into basic grayscale modulation and extended grayscale modulation. The basic gray level refers to the gray level generated by the reference time $\tau_{0}$ as the minimum modulation period. If the refresh rate of the LED display is $f_{\text {frame }}$, the period of one frame of data is $T_{\text {frame }}$ :

$$
T_{\text {frame }}=\frac{1}{f_{\text {frame }}} .
$$

The frame period includes the maximum number of time slices $n_{\text {int }}$ :

$$
n_{\mathrm{int}}=\frac{T_{\text {frame }}}{\tau_{0}} .
$$

Thus, the basic gray level number $N_{\text {int }}$ is:

$$
N_{\text {int }}=f i x\left(\log _{2} n_{\text {int }}\right) .
$$

Expanding the gray level means that the gray level is generated with the minimum response pulse width $\tau_{o e}$ as the minimum modulation period within the reference time $\tau_{0}$. Thus, the reference time contains 20. The number is $n_{f r a}$ for:

$$
n_{\text {fra }}=\frac{\tau_{0}}{\tau_{o e}} .
$$

This will result in an expanded gray level number of $N_{\text {fra }}$ :

$$
N_{f r a}=f i x\left(\log _{2} n_{f r a}\right) .
$$

The LED display gray level $n$ is a superposition of the basic gray level and the extended gray level:

$$
n=N_{\text {int }}+N_{\text {fra }} .
$$

The performance of the LED display control system is closely related to the selected driver chip. The maximum scan clock and minimum response pulse width are directly affected by the parameters of the driver chip itself. Table 3 lists the driver chip parameters of four different manufacturers. It can be seen from Table 3 that the scan clock of the driver chip is below $35 \mathrm{MHz}$. On the one hand, the excessive scan clock may exceed the limit speed of the driver chip itself, and on the other hand, the PCB layout and layout of the display module are also higher. The minimum response pulse width is above 30 ns. Too small pulse width time will cause the driver chip to not respond correctly. Therefore, the driver chip can limit the grayscale parameters to:

$$
\left\{\begin{array}{l}
\tau_{o E} \geq 30 \mathrm{~ns} \\
f_{c l k} \leq 35 \mathrm{MHz}
\end{array} .\right.
$$

The minimum response pulse width of the driver chip is the effective width at which channel can be opened under the condition that the linearity of all channel output currents can be maintained. If the given pulse width is less than the minimum response pulse width, the channel output does not respond or the response is non-linear. If the given pulse width is greater than or equal to the minimum response pulse width, the channel output can respond correctly. Due to the unevenness of the chip manufacturers, the technical specifications given by the manufacturer are also incorrect. Therefore, after selecting the driver chip, the minimum response pulse width of the driver chip must be tested, and the value used is slightly larger than the test value (5-10) ns. It to some extent offsets the inconsistency of the chip pulse width response. The driver chip on the LED display module adopts TI's TLC59282, the serial data input is given a high level; the clock is $10 \mathrm{MHz}$; the latch is a periodically changing pulse with a period of $13 \mathrm{~ns}$ and a high level width of $200 \mathrm{~ns}$. The given response pulse width is $40 \mathrm{~ns}$. These are all implemented by FPGA programming. According to the technical ma- 
Table 3. Parameters of Small Driver Chip

\begin{tabular}{|l|c|c|c|c|}
\hline \multicolumn{1}{|c|}{ Driver chip model } & MB15024 & TCL59282 & SMI6126 & SD16739 \\
\hline Minimum response pulse width, ns & 70 & 30 & 30 & 30 \\
\hline Maximum scan clock, MHz & 25 & 35 & 25 & 30 \\
\hline
\end{tabular}

nual, $\tau_{o e}$ is 40 ns as the pre-test object, and the channel output response of 10 groups of chips is tested. The response result is up to $41.8 \mathrm{~ns}$, deviating from the given pulse width of 4.5070, and other response results are greater than the given pulse width of 40 ns. This deviation is mainly caused by the chip itself and can be corrected by the late point-by-point uniform correction technique.

It is analyzed by formula (10) and formula (15) and their associated formulas. For each level of basic gray level, the number of reference time slices needs to be increased by 2 times. The methods and analysis can be taken as follows: the scan clock is doubled, but it cannot be breaking through the limitations of the drive chip itself on the clock frequency. Halving the length of the transfer or halving the number of scan lines will result in a reduction in the control range of the control system. The refresh rate is halved, which affects the visual effect. In general, the increase in the reference gray level will be limited by the performance of the device, and will also bring other performance degradation. The method and analysis that can be taken for each level of gray level expansion is as follows below. The minimum response pulse width is halved, but it cannot break the minimum time that the driver chip itself can respond. The scan clock is halved or the transfer length is doubled, which results in a halving of the basic gray level, which is of no practical significance. In the gradation modulation, after the parameters are preferably determined, the gradation control can be performed. Here, a time slice matching algorithm is adopted, that is, the time slice occupied by the high-weight data bits is split, and the low-weight data bit time slice is mixed and modulated in combination with the case that the total number of time slices is the same. In order to better understand the time slice split, when the gray modulation model parameters $n=14, m=9$, the latch sequence and the blanking sequence are respectively:

$$
L_{x}=\left\{\begin{array}{l}
1, x<9 \\
2^{x-9}, x \geq 9
\end{array}(0 \leq x<14)\right.
$$

\section{RESULT ANALYSIS AND DISCUSSION}

When the minimum pulse width is selected as $40 \mathrm{~ns}$ and the refresh rate is $600 \mathrm{~Hz}$, the relationship between the gray level, the shift length, and the relationship between the scan clocks is as shown in Fig.1, in which the scan clock is changed between 1 and $30 \mathrm{MHz}$. The storage length varies between 16 and 512 .

As can be seen from Fig. 1, the gray level varies between 14 bit and 15 bit, 14 bit is mostly, 15 bit is less, and the distribution is concentrated on several strips of radiation. The upper right picture shows the amplification of the low-speed clock area. It can be seen that when the transfer length is less than 200, the fluctuation of the gray level distribution fluctuates greatly. In the area above 200, the gray level changes relatively smoothly. The lower left image is an enlargement of the area with a small transfer length. The 16-64 area is selected. It can be seen that the gray level of the area changes the same as the upper left picture.

Fig. 2 is a state in which the gradation level exhibits disordered fluctuations in the low-speed clock region where the transfer length is small. During the whole modulation period, the time slice matching algorithm realizes that the LED lights up according to a certain rule, so that the LED does not appear bright or off for a long time, thereby achieving

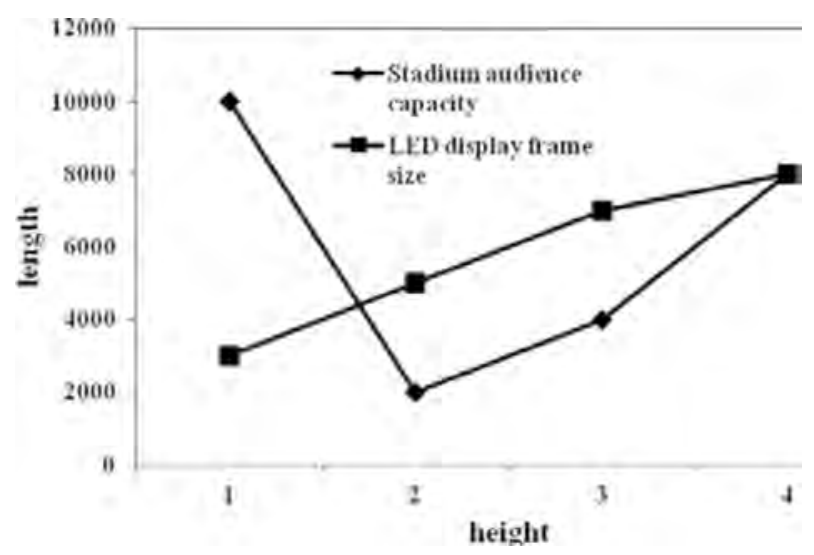

Fig.1. Determination of the size of the frame of the stadium LED display 


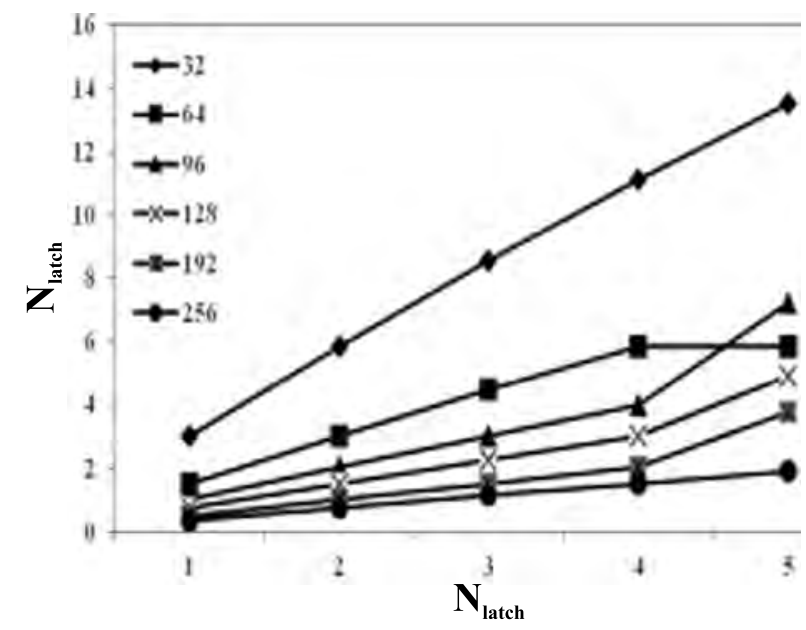

Fig.2. Proportion of brightness loss within the reference time

the purpose of reducing the flickering of the display screen. In this section, based on the characteristics of LED gray-scale modulation, an evaluation strategy based on the flash frequency factor is proposed to qualitatively evaluate the flickering of the screen. This method evaluates different time slice combinations and belongs to the category of gray-scale modulation algorithms. The human eye produces a flickering effect on the rapidly changing optical signal. Since the LED is off and on, when the LED changes frequency is not fast enough, the human eye can perceive a noticeable flickering feeling. When the frequency of change is high, due to the visual inertia of the human eye, the observer will no longer feel flicker, and usually will not cause flicker, that is, the frequency at which the human eye can feel a stable picture, called the critical flicker frequency. The critical flicker frequency of the human eye is related to many factors: the brightness of the blinking picture; the higher the brightness is, the higher the critical flicker frequency is. The amplitude of the flicker: the larger the amplitude is, the more obvious the flicker is perceived by the human eye. When the amplitude is smaller than the brightness that can be resolved by the human eye, the observer will not feel the flicker. Observation time, short-term observation is not obvious to the flicker, and it is easier to feel the flicker when observed for a long time.

For the area of the image, the larger the angle of view is observed by the human eye, the higher the critical flicker frequency is; the frequency at which the LED light is on and off at the same brightness and refresh rate. With the rapid development of displays, people are increasingly demanding displays.

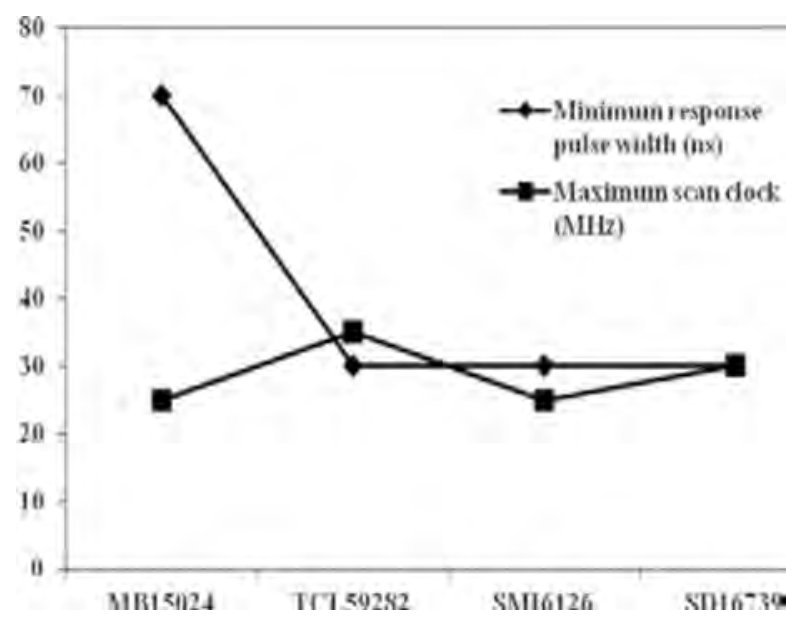

Fig.3. Parameters of small driver chip

The degree of flickering of the screen has certainly become a hot issue of concern. Fig. 3 reflects the subjective evaluation of the display effects of different types of displays under different conditions. The observation results show that when the outdoor display brightness is greater than $4000 \mathrm{~cd} / \mathrm{m}^{2}$, it is better to use a refresh rate above $400 \mathrm{~Hz}$; when the brightness is less than $4000 \mathrm{~cd} / \mathrm{m}^{2}$, it is better to use a refresh rate above $240 \mathrm{~Hz}$. The degree of flicker is not enough to measure by the refresh rate, because in the case of the same refresh rate, the difference in the gray-scale modulation algorithm is different. The time $T$ occupied by arbitrarily selecting two adjacent time slices in the LED working waveform is regarded as one sub-period. Among them, the high level indicates that the LED display is lit, the time is $T_{1}$, and the low level indicates that the LED is off and the time is $T_{Z}$. There are many factors affecting the flash factor. Here are the effects of different modulation factors $m$, different matching parameters $g$ and different matching combinations on the flash factor. According to the above analysis method, other parameters can be analogized, such as the influence of different gamma on the flash factor. Using the evaluation strategy of the flash frequency factor, the design parameters can be relied upon to avoid artificial subjective speculation.

\section{CONCLUSION}

A method for selecting the preferred gray control parameters suitable for engineering applications is provided. According to the gray modulation model, the gray level modulation is divided into basic gray level modulation and extended gray level modulation, and a preferred scan clock is given. The 
selection method and the concept of scan clock redundancy are given. The maximum scan clock redundancy can be used as an indicator to judge the hardware performance of the control system. In addition, on this basis, the optimization method of LED display control parameters is summarized, which provides sufficient theoretical guidance for the design of LED display control system. A time slice matching algorithm is proposed to improve the stability of the displayed image and reduce the stroboscopic effect. After determining the optimization parameters, the optimization parameters can be used to implement the gray-scale modulation algorithm to achieve the purpose of optimizing performance. Here, through the time slice matching algorithm, the arrangement of the time slices can be relied upon to make the LEDs turn on and off more evenly, thereby achieving the purpose of reducing the flickering of the display screen. At the end of the paper, an evaluation strategy based on the flash frequency factor is also presented, which can be used to judge the results of time-scale gray-scale modulation, which provides an important basis for the update and improvement of the algorithm.

\section{REFERENCES:}

1. Ahn H A, Hong S K, Kwon O K. An Active Matrix Micro-Pixelated LED Display Driver for High Luminance Uniformity Using Resistance Mismatch Compensation Method. IEEE Transactions on Circuits and Systems II: Express Briefs, 2018. V65, \#6, pp.724-728.
2. Chun J, Lee M. Developing a SEIL (Smart Enjoy Interact Light) bag utilizing LED display. International Journal of Clothing Science and Technology, 2016. V28, \#2, pp.233-253.

3. Delabrida S, D'Angelo T, Oliveira R A R, et al. Wearable HUD for Ecological Field Research Applications. Mobile Networks and Applications, 2016. V21, \#4, pp.677-687.

4. Bai Y, Welk G J, Nam Y H, et al. Comparison of consumer and research monitors under semistructured settings. Medicine \& Science in Sports \& Exercise, 2016. V48, \#1, pp.151-158.

5. Ellis B J, Volk A A, Gonzalez J M, et al. The meaningful roles intervention: An evolutionary approach to reducing bullying and increasing prosocial behavior. Journal of research on adolescence, 2016. V26, \#4, pp.622-637.

6. Roy R, Hebden L, Kelly B, et al. Description, measurement and evaluation of tertiary-education food environments. British Journal of Nutrition, 2016. V115, \#9, pp.1598-1606.

7. Konstantinidis E I, Billis A S, Mouzakidis C A, et al. Design, implementation, and wide pilot deployment of FitForAll: an easy to use exergaming platform improving physical fitness and life quality of senior citizens. IEEE journal of biomedical and health informatics, 2016. V20, \#1, pp.189-200.

8. Xu, X., Xie, L., Li, H., \& Qin, L. Learning the route choice behavior of subway passengers from AFC data. Expert Systems with Applications, 2018. \#95, pp. 324-332.

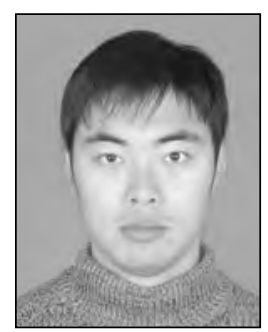

\section{Jing LIU,}

Master of Physical Sport Training, Lecturer. Graduated from the Northwest Normal University in 2004. Worked in BeiHang University. His research interests include Physical education and Sports training, Sports Humanity Education 